\title{
Wearable Wireless Power Transfer using Direct-Write Dispenser Printed Flexible Coils
}

\author{
Mahmoud Wagih, Graduate Student Member, IEEE, Abiodun Komolafe, and Bahareh Zaghari \\ School of Electronics and Computer Science, University of Southampton, \\ mahm1g15@ecs.soton.ac.uk
}

\begin{abstract}
Direct-write dispenser printing represents a simple solution to rapid flexible and printed electronics prototyping and low-volume manufacturing. This work presents the design, fabrication and evaluation of magnetic resonance wireless power transfer (WPT) coils fabricated using dispenser printing. A double-sided inductor is designed and printed on a flexible polyimide substrate using a commercial dispenser printer. The dispenser printer is used to realize the single-sided coils which are heat pressed to form double-sided inductors using a conductive epoxy via. It is demonstrated that the proposed double-sided coils achieve over $53.8 \%$ higher quality factor than a single-sided coil, and $67 \%$ higher inductance than two series-connected coils. The coils are tuned to resonate at $6.78 \mathrm{MHz}$ using lumped-matching. The proposed coils achieve a peak WPT efficiency of $50 \%$.

Index Terms-Additive Manufacturing, Coils, E-textiles, Dispenser Printing, Inductor, Internet of Things, Wireless Power Transfer
\end{abstract}

\section{INTRODUCTION}

Additive manufacturing is increasingly seen as an enabling method of realizing flexible, wearable and organic electronic systems for the emerging unobtrusive Internet of Things (IoT) market [1]. Wireless Power Transfer (WPT) and energy harvesting represent potential candidates for powering and re-charging flexible electronics sustainably without the need for a physical connection [2]. Recent work has shown the possibility to realize electromagnetic and Radio Frequency (RF) energy harvesters and power transfer units based on printed and flexible electronics [3].

Dispenser printing allows "direct-writing" of a 2D circuit design on a flexible or a rigid substrate. Dispenser printing has been previously utilized to fabricate energy harvesters [4], textile displays [5], and antenna prototypes [6]. Compared to screen-printing and photolithography, dispenser printing allows a faster-turnaround as it does not require a dedicated screen or mask.

Magnetic Resonant (MR) WPT enables improved spatial freedom and coil-separation over inductive coupling, while theoretical maximum high WPT efficiencies of over $90 \%$. Multiple flexible and wearable MR-WPT have been presented using flexible printed circuit boards (PCB) [7], embroidered textile coils [8], and adhered copper foils to flexible and textile substrates [9], [10]. However, the realization of MR-WPT systems using dispenser-printed flexible coils has not been previously investigated.

This work was supported by the U.K. Engineering and Physical Sciences Research Council (EPSRC) under Grant EP/P010164/1

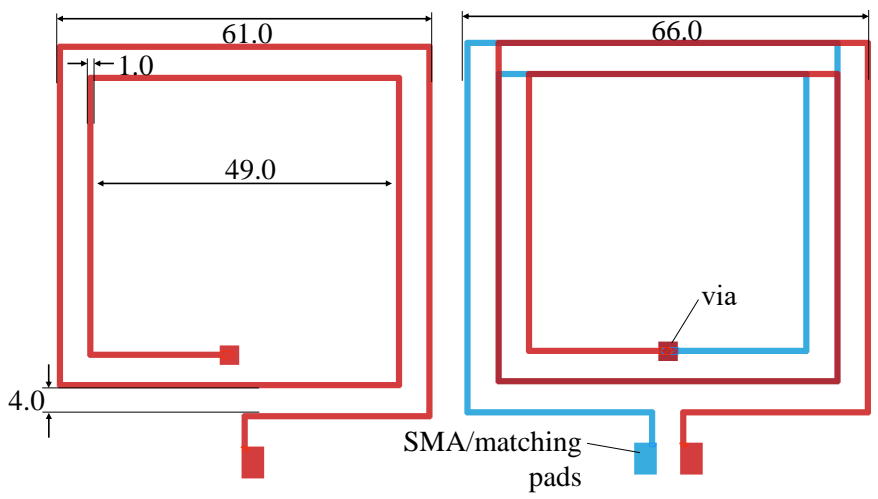

Fig. 1. Dimensions (in $\mathrm{mm}$ ) of the proposed coil. Left: top layer (singlesided), right: double-sided coil.

This work utilizes a commercial dispenser printer to realize double sided MR-WPT coils on a thin and flexible substrate. It is shown that using the proposed double-sided geometry it is possible to improve the coil's quality factor by $53.8 \%$ compared to single-sided coils. The efficiency of the WPT link is investigated and it is shown that with a coil design optimized for printing, it is possible to achieve WPT efficiencies up to $50 \%$ and less than $2 \mathrm{~dB}$ degradation in performance when operating on-body.

\section{CoIl Design And FABRicAtion}

\section{A. Coil Design}

The key to high WPT efficiency is a high coil Quality (Q)-factor (1), this is achieved using coils of minimal series resistance $R$ and higher self inductance $L$ [7].

$$
Q=\frac{\omega L}{R}
$$

When utilizing printed conductors, achieving conductivity similar to copper sheets or litz wires is not possible due to the higher surface resistivity (in order of $1 \Omega /$ square) and the high surface roughness of over $10 \mu \mathrm{m}$. Therefore, increasing the number of turns or the coil's radius to improve the inductance will inevitably increase the series resistance. To increase the inductance of the coils a double-sided inductor is utilized. This allows reducing the number of turns, and hence the overall geometry of the whole WPT system. A double-sided two-turn coil of 1-mm trace width and 4-mm pitch is proposed. Fig. 1 shows the geometry of the coil. 
(a)

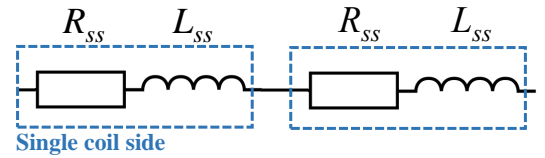

(b)

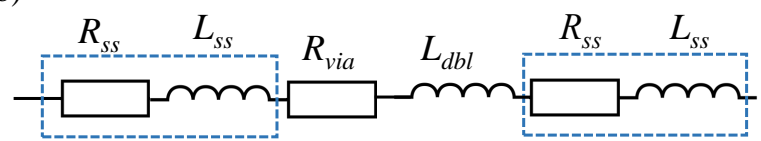

Fig. 2. Equivalent circuit of the (a) two single-sided (ss) inductors connected in series and (b) double-sided (dbl) inductor showing the additional inductance and resistance due to increased turns and the via.

The designed coils can be modeled as a series inductor and a parasitic resistor (Fig. 2-a), due to the series resistance of the conductive traces. While additional coil connections in series could be utilized to increase the inductance, the series resistance will increase reducing the coils $Q$. On the other hand, when the proposed double-sided geometry is utilized, an additional inductance term $\left(L_{d b l}\right)$ is introduced due to the increase overall number of turns, as observed in Fig. 1, in addition to the inherent self-inductance of each of the coil's sides. Therefore, despite the increased series resistance introduced by the via $\left(R_{v i a}\right)$, the improvement in the coil's inductance could be utilized to increase the coil's Q-factor.

The substrate chosen in this work is polyimide, due to its improved thermal and mechanical reliability while not compromising on flexibility, and the overall "low-cost" designgoal of printed IoT devices.

\section{B. Direct-Write Dispenser Printing}

A commercial dispenser printer (Voltera V-one) has been used to print the conductive traces onto the flexible polyimide substrate. $75 \mu \mathrm{m}$ polyimide sheets have been adhered to a rigid surface, an alumina tile, using Kapton tape to keep the substrate planar during printing. The coil CAD drawing is loaded to the printer where a printing map is automatically generated. The silver ink is then jetted onto the substrate following thickness measurements to prevent a nozzle crash.

After the ink is jetted, the substrate is transported to a hotplate to cure the ink. A standard soldering hot-plate is used to cure the ink at $220^{\circ} \mathrm{C}$ for 30 minutes. While the Voltera printer allows curing the boards on its own hot-plate with the conductor facing the hot plate, a standard hot-plate is used to provide the heat from below to prevent smearing the ink on the flexible substrate.

The process is repeated on a different polyimide sheet to fabricate the coil's bottom layer. After both layers are cured, heat-pressing with $100 \mu \mathrm{m}$ thermo-plastic polyurethane adhesive sheet is used to realize the double-sided coil. The coils have been pressed at $190^{\circ} \mathrm{C}$ for 1 minute. A hole is then drilled and filled with conductive silver-loaded epoxy to create the via connecting both sides of the coil. Fig. 3 shows the fabrication steps of the coil from conductor printing to the complete structure with the via. The epoxy has been cured at $120^{\circ} \mathrm{C}$ for 5 minutes. (a)

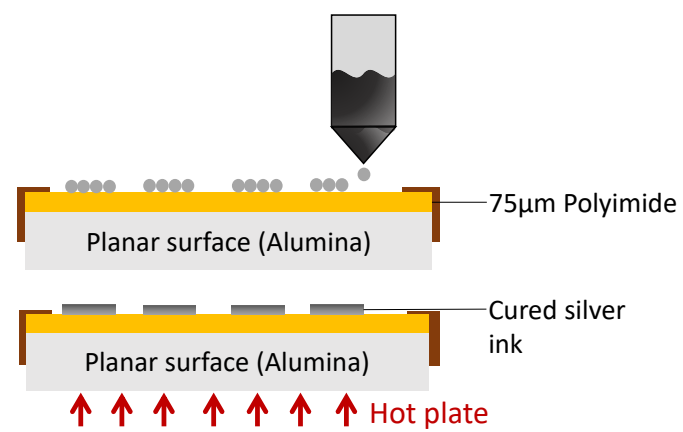

(c)

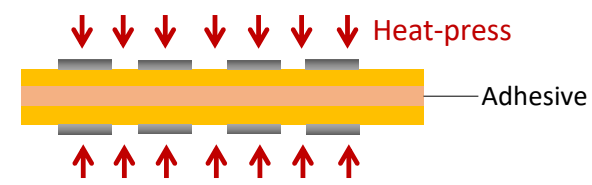

(d)

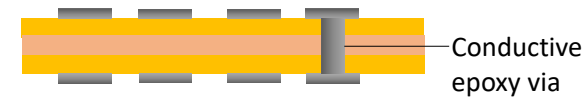

Fig. 3. Fabrication steps of the double-sided WPT coil: (a) silver ink printing on a planar polyimide substrate, (b) silver ink curing on a hot plate, (c) thermal-pressing of the single-sided top and bottom coils, (d) the assembled double-sided coil showing the conductive epoxy via.

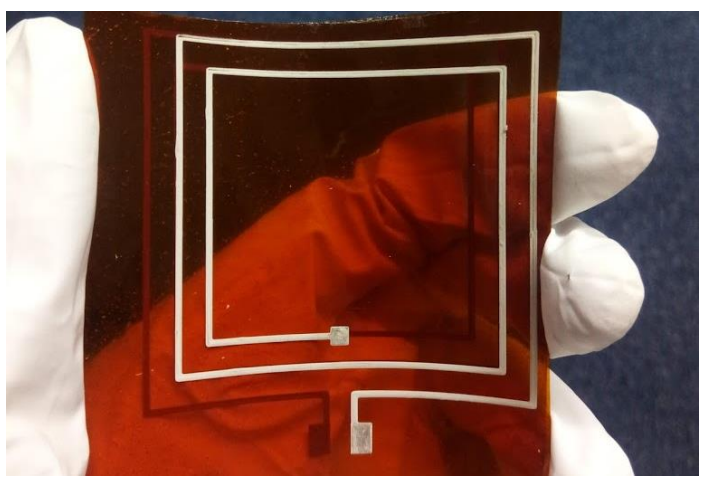

Fig. 4. Photograph of the fabricated double-sided coil.

\section{COIL PARAMETERS}

An impedance analyzer (Wayne Kerr 6500B) has been used to measure the electrical properties of the fabricated coil. This is paramount to tuning the coils to certain frequencies, calculating the Q-factor, and evaluating different fabrication methods [11]. The parameters of individual coil sides has been measured (single-sided) as well as the parameters of the double-sided coil. Table I shows the measured electrical characteristics of the single and double-sided coils.

TABLE I

MEASURED COIL ELECTRICAL PARAMETERS AT $6.78 \mathrm{MHz}$

\begin{tabular}{|c|c|c|c|}
\hline Coil & $\mathrm{L}(\mu \mathrm{H})$ & $\mathrm{R}(\Omega)$ & $6.78 \mathrm{MHz}$ Q-factor \\
\hline Single-layer & 0.59 & 3.95 & 1.01 \\
\hline Double-sided & 1.97 & 8.6 & 1.55 \\
\hline
\end{tabular}




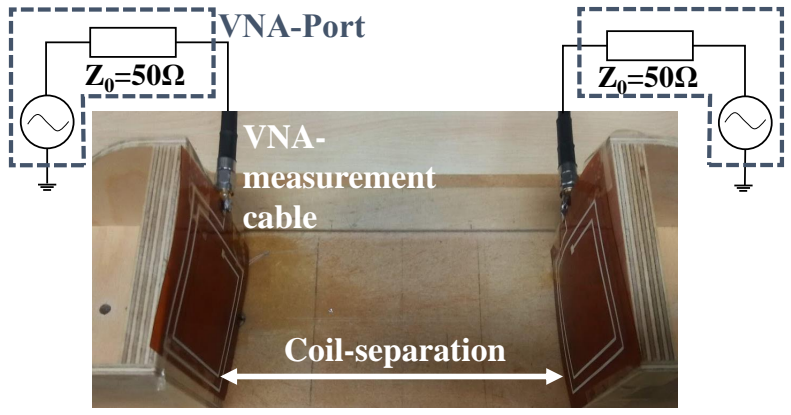

Fig. 5. Test setup of the printed coils using the VNA.

The double-sided coil, having a Q-factor of 1.55 as opposed to the single-sided 1.01 Q-factor, demonstrates the benefits of utilizing double-sided coils for additively-manufactured WPT coils.

\section{MR-WPT TESTING AND Evaluation}

The coils have been matched using lumped capacitors to resonate at $f_{r}=6.78 \mathrm{MHz}$, the Industrial Scientific and Medical band (ISM-band) used by the Airfuel WPT alliance. The value of the tuning capacitors has been calculated using (2). The capacitor for tuning used is a $270 \mathrm{pF}$ ceramic capacitor.

$$
C=\frac{1}{4 \pi^{2} f_{r}^{2} L}
$$

SMA coaxial connectors have been soldered to the coils, using a low-temperature solder not to damage the traces, to allow measurement of the WPT efficiency. A ZVB4 Vector Network Analyzer (VNA) has been used to measure the twoport s-parameters of the coils for different test conditions. The WPT efficiency can be calculated using the power forward transmission from the measured s-parameters $\eta_{W P T}=\left|S_{21}\right|^{2}$.

The coils, mounted on wooden test fixtures, have been positioned at variable separations to measure the s-parameters of the coils. Fig. 5 shows the experimental test setup of the coils using. Fig. 6 shows the two-port s-parameters, showing the voltage forward transmission $\left(\mathrm{S}_{21}\right)$ and reflection coefficient $\left(\mathrm{S}_{11}\right)$ at $1 \mathrm{~cm}$ separation 7 . The forward voltage transmission and the WPT efficiency of the coils at variable separation is shown in Fig. 7.

In wearable applications, such as smart cycling gloves [8], the coils are expected to operate under close-proximity with the human body. In the case of using MR-WPT to transfer power from an energy-harvesting bicycle to a glove, the coils need to maintain high efficiency in tight coupling $(<2$ $\mathrm{cm}$ separation) and on-body. The s-parameters of the coils positioned on- and off-hand placed at $2 \mathrm{~cm}$ separation are shown in Fig. 8, demonstrating under $2 \mathrm{~dB}$ degradation in performance when operating on-body.

\section{CONCLUSION}

This paper presented direct-written dispenser printed flexible WPT coils for flexible IoT and wearable applications. The proposed double-sided printed coils achieve $53.7 \%$ higher

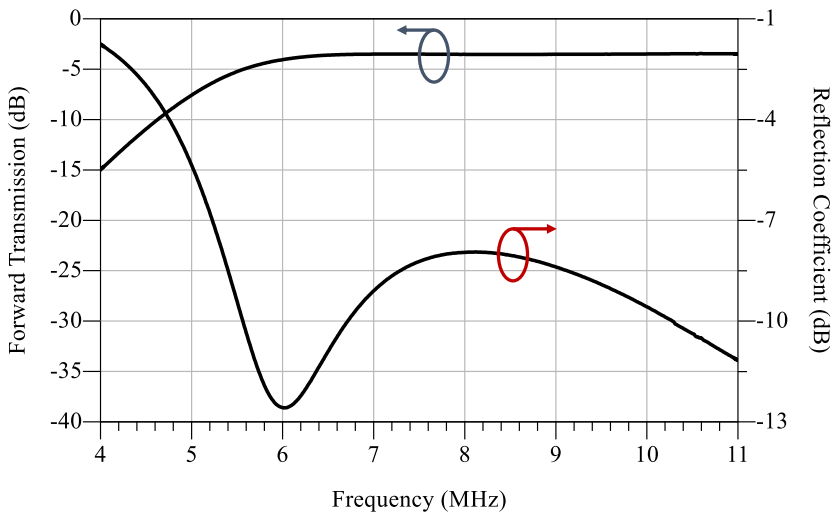

Fig. 6. Measured two port s-parameters of the coils at 1-cm separation showing a peak $S_{21}=-5.6 \mathrm{~dB}$.

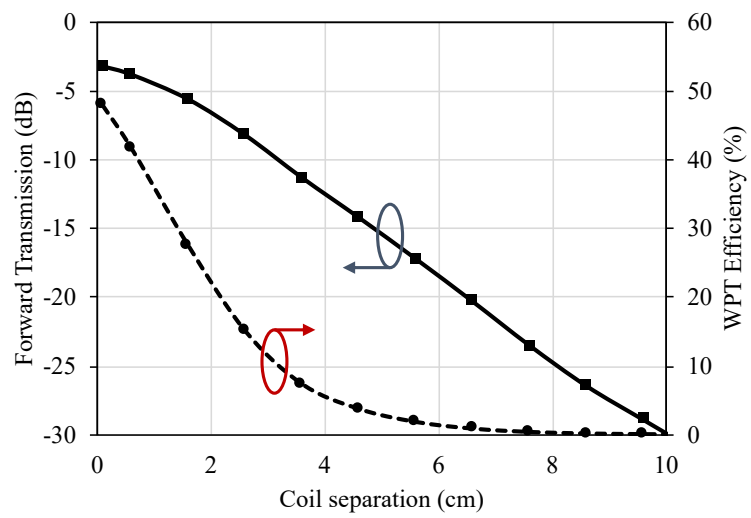

Fig. 7. Measured forward transmission and WPT efficiency of the printed coils at variable coil separation.

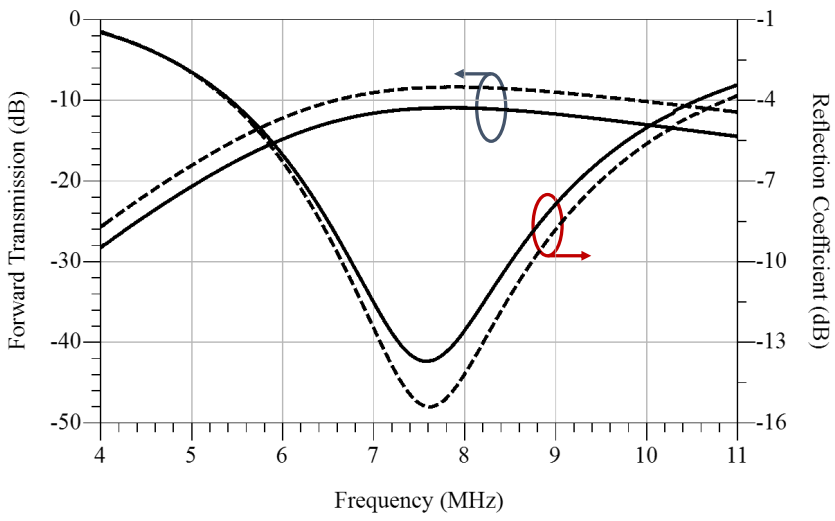

Fig. 8. Measured two port s-parameters of the coils at 2-cm separation inspace (dashed) and on-hand (solid).

quality factor compared to a single-sided printed coil of the same geometry and up to 50\% WPT efficiency due to their double-sided structure enabling higher WPT efficiency without increasing the coils' dimensions. This demonstrates the feasibility of utilizing low-cost dispenser printing to realize WPT systems for next generation flexible and printed electronics. 


\section{REFERENCES}

[1] S. Khan, L. Lorenzelli, and R. S. Dahiya, "Technologies for printing sensors and electronics over large flexible substrates: A review," IEEE J. Sensors, vol. 15, no. 6, pp. $3164-3185,2015$.

[2] J. Garnica, R. A. Chinga, and J. Lin, "Wireless power transmission: From far field to near field," Proceedings of the IEEE, vol. 101, 6, pp. $1321-1331,2013$.

[3] S. A. Nauroze, J. G. Hester, B. K. Tehrani, W. Su, J. Bito, R. Bahr, J. Kimionis, and M. M. Tentzeris, "Additively Manufactured RF Components and Modules: Toward Empowering the Birth of Cost-Efficient Dense and Ubiquitous IoT Implementations," Proc. IEEE, vol. 105, no. 4, pp. $702-722,2017$.

[4] A. Chen, D. Madan, P. K. Wright, and J. W. Evans, "Dispenserprinted planar thick-film thermoelectric energy generators," Journal of Micromechanics and Microengineering, vol. 21, 2011.

[5] Y. Wei, X. Wang, R. Torah, and J. Tudor, "Dispenser printing of electrochromic display on textiles for creative applications," Electronics Letters, vol. 53 (12), pp. 779 - 781, 2017.

[6] M. Wagih, "Direct-Write Dispenser Printing for Rapid Antenna Prototyping on Thin Flexible Substrates," in 2020 European Conference on Antennas and Propagation (EuCAP), 2020.

[7] T. Imura and Y. Hori, "Maximizing air gap and efficiency of magnetic resonant coupling for wireless power transfer using equivalent circuit and neumann formula," IEEE Transactions on Industrial Electronics, vol. 58 , no. 10 , pp. 4746 - 4752, 2011.

[8] M. Wagih, A. Komolafe, and B. Zaghari, "Dual-receiver wearable 6.78 mhz resonant inductive wireless power transfer glove using embroidered textile coils," IEEE Access, vol. 8, pp. 24630 - 24642, 2020.

[9] S. H. Kang, V. T. Nguyen, and C. W. Jung, "Analysis of mr-wpt using planar textile resonators for wearable applications," IET Microwaves, Antennas \& Propagation, vol. 10, 14, pp. 1541 - 1546, 2016.

[10] K. Bao, C. L. Zekios, and S. V. Georgakopoulos, "A wearable wpt system on flexible substrates," IEEE Antennas and Wireless Propagation Letters, vol. 18, 5, pp. 931 - 935, 2019.

[11] N. J. Grabham, Y. Li, L. R. Clare, B. H. Stark, and S. P. Beeby, "Fabrication techniques for manufacturing flexible coils on textiles for inductive power transfer," IEEE Sensors Journal, vol. 18, 6, pp. 2599 2606, 2018. 